\title{
Investigation of the Mitochondrial Haplogroups in A Selective Population of Isfahan Province
}

\author{
Elham Iranpour ${ }^{1}$, Elmira Sanjari ${ }^{2}$, Hasan Bahmani ${ }^{3}$, Asmaolhosna Amini ${ }^{3}$, Hasan Abbasi ${ }^{4}$, Mahmoud Tavallaie ${ }^{3}$ \\ and Massoud Houshmand ${ }^{2 *}$ \\ ${ }^{1}$ Nour Danesh Institute of Higher Education, Iran
}

${ }^{2}$ National Institute of Genetic Engineering and Biotechnology, Iran

${ }^{3}$ Nour Human Genetics Research Institute, Iran

${ }^{4}$ Environment Department, Isfahan University, Iran

Submission: December 30, 2017; Published: April 25, 2018

*Corresponding author: Massoud Houshmand, National Institute of Genetic Engineering and Biotechnology, Tehran, Iran,

Email: massoudh@nigeb.ac.ir

\begin{abstract}
In order to investigate the mitochondrial haplogroups in a selective population of Isfahan province, 96 unrelated men in Isfahan province with at least two generations in this province were token blood. Then DNA was extracted and quality assessed. 25 coding SNPs defining the major haplo groups that occur in Africa, Western Eurasia and Eastern Eurasia were selected and combined into two multiplex genotyping assays. Each one consisting of a PCR step and a SNaPShot step. Then for support of our detective haplo groups by SNaPShot system, D-Loop region was sequenced in some samples. : In this province Western Eurasian haplo groups were predominant. Haplo group U and H $(22 \%, 21 \%)$ and then T, J and U8b (11\%, 10\% and 7\%) had the most frequency. Eastern Eurasian haplo groups (C, D, R9, R11, M and N) were present at a lower frequency and a North African haplo group (M1) at frequency of $1 \%$.
\end{abstract}

Keywords: mtDNA; SNP; Haplo group; Isfahan province

\section{Introduction}

Human mitochondrion has a DNA independent of nucleus DNA called mtDNA. mtDNA is a dabble strand circular molecular that has some special feathers that make it useful in human evolution studies. It has maternal inheritance [1], higher rate of mutation than nuclear DNA [2], high copy numbers and lack or recombination[3]. Due to these special feathers, mtDNA are widely used as a useful tool in studies like population history, medical genetics, genetic genealogy, and genetic forensic. Analyzing mtDNA patterns in modern human use for tracing genetics journey of early women. By mtDNA studies researchers can trace a targeted maternal ancestry along time and defining maternal ancestor in a selective population.

A haplogroup is a genetically group of population that has common ancestors in maternal/paternal lines. Mutated nucleotides in mitochondrial genome are transferred as polymorphisms from mother to boy and girl children. The whole polymorphisms in an individual mtDNA is called mitochondrial haplotype. Varies haplo types in different ancestries create branches of mtDNA phylogenic tree and consequently aggregation of haplo types in this tree creates clusters as haplo group [4]. mtDNA phylogenic tree is classified in four macro haplo groups named L, M, N and R. each one has some haplo groups and each haplo group has some sub haplo groups.

mtDNA and Y chromosome haplo groups studies are useful in cases like investigation on human colonization patterns from different continents and understanding origin and genetic structure of different populations.

The Purpose of this study is defining of each haplo group frequency in a selective population of Isfahan province, a central province in Iran.

\section{Material and Methods}

96 unrelated men inhabited in Isfahan province with at least two generations in this province were token blood, based on the number of population of each city. Then DNA was extracted by RGDE method [5] and quality assessed by nano photometer and electrophoresis. We selected 25 coding SNPs [6] defining 32 of the major haplo groups that occur in Africa, Western Eurasia and Eastern Eurasia (Figure 1). SNPs combined into two multiplex genotyping assays. Each one consisting of a PCR step and a SNaPShot step. 


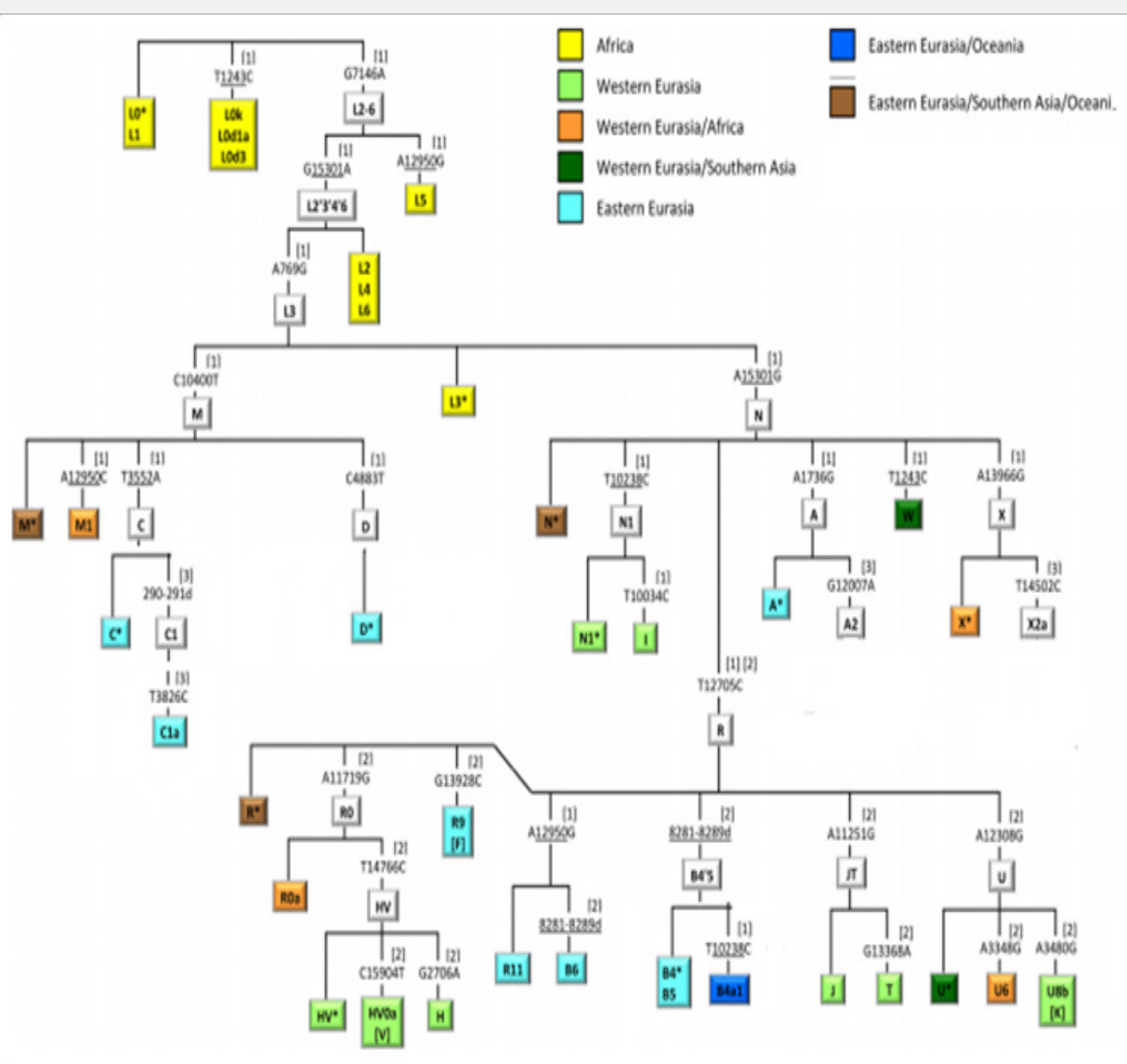

Figure 1: Targeted mtSNPs with geographic haplogroup classification

Table 1: Multiplex 1 Primers details

\begin{tabular}{|c|c|c|c|c|c|c|c|c|c|}
\hline \multirow[b]{2}{*}{ Site } & & \multicolumn{2}{|c|}{ PCR amplification } & & \multicolumn{2}{|c|}{ Single-base extension } & \multirow[b]{2}{*}{ Length } & \multirow[b]{2}{*}{ Orientation } & \multirow[b]{2}{*}{$\begin{array}{l}\text { Alleles } \\
\text { (dye) }\end{array}$} \\
\hline & & $\begin{array}{c}\text { Primer } \\
\text { sequences } \\
\left(5^{\prime}-3^{\prime}\right)\end{array}$ & Concentration & $\begin{array}{c}\text { Amplicon } \\
\text { size (bp) }\end{array}$ & $\begin{array}{c}\text { Primer } \\
\text { sequence } \\
\left(5^{\prime}-3^{\prime}\right)\left(5^{\prime}\right. \\
\text { aspecific tail } \\
\text { in lowercase } \\
\text { italics) }\end{array}$ & Concentration & & & \\
\hline & & (pM) & & & (pM) & (nt) & & & \\
\hline \multirow[t]{2}{*}{769} & $\mathrm{~F}$ & $\begin{array}{c}\text { ACATC } \\
\text { ACCCCAT } \\
\text { AAACAA } \\
\text { ATAGG }\end{array}$ & 1 & 158 & $\begin{array}{l}\text { act(gact)10 } \\
\text { CGTTTT } \\
\text { GAGCTG } \\
\text { CATTG }\end{array}$ & 2 & 60 & $\mathrm{R}$ & $\begin{array}{l}\text { A (red), G } \\
\text { (yellow) }\end{array}$ \\
\hline & $\mathrm{R}$ & $\begin{array}{c}\text { AGCGT } \\
\text { TTTGAGC } \\
\text { TGCATTG }\end{array}$ & 1 & & & & & & \\
\hline \multirow[t]{2}{*}{1243} & $\mathrm{~F}$ & $\begin{array}{c}\text { AATCGAT } \\
\text { AAACCC } \\
\text { CGATCAA }\end{array}$ & 0.04 & 93 & $\begin{array}{c}\text { actgact CGATC } \\
\text { AACC } \\
\text { TCACC } \\
\text { ACC }\end{array}$ & 0.15 & 24 & $\mathrm{~F}$ & $\begin{array}{c}\mathrm{C} \text { (yellow) } \\
\mathrm{T} \text { (red) }\end{array}$ \\
\hline & $\mathrm{R}$ & $\begin{array}{l}\text { TGCGCT } \\
\text { TACTT } \\
\text { TGTAG } \\
\text { CCTTC }\end{array}$ & 0.04 & & & & & & \\
\hline
\end{tabular}




\begin{tabular}{|c|c|c|c|c|c|c|c|c|c|}
\hline 1736 & F & $\begin{array}{c}\text { GCTAAA } \\
\text { CCTAGC } \\
\text { CCCAA } \\
\text { ACC }\end{array}$ & 0.2 & 106 & $\begin{array}{c}\text { t(gact)2gac } \\
\text { TCAAT } \\
\text { TTCTA } \\
\text { TCGCC } \\
\text { TATAC } \\
\text { TTTAT }\end{array}$ & 0.15 & 37 & $\mathrm{R}$ & $\begin{array}{l}\text { A (red), G } \\
\text { (yellow) }\end{array}$ \\
\hline & $\mathrm{R}$ & $\begin{array}{c}\text { CTAT } \\
\text { TGCGC } \\
\text { CAGGTT } \\
\text { TCAAT }\end{array}$ & 0.2 & & & & & & \\
\hline \multirow[t]{2}{*}{3552} & F & $\begin{array}{c}\text { CGCTGA } \\
\text { CGCCA } \\
\text { TAAAA } \\
\text { CTCT }\end{array}$ & 4 & 128 & $\begin{array}{l}\text { actgact } \\
\text { AGGGGG } \\
\text { GTTCATA } \\
\text { GTAGAAG }\end{array}$ & 4 & 27 & $\mathrm{R}$ & $\begin{array}{c}\text { A (red), C } \\
\text { (blue), T } \\
\text { (green) }\end{array}$ \\
\hline & $\mathrm{R}$ & $\begin{array}{l}\text { GTATG } \\
\text { GGGAG } \\
\text { GGGG } \\
\text { GTTC }\end{array}$ & 4 & & & & & & \\
\hline \multirow[t]{2}{*}{4883} & F & $\begin{array}{c}\text { TGAC } \\
\text { ATCC } \\
\text { GGCC } \\
\text { TGCTT }\end{array}$ & 0.075 & 114 & $\begin{array}{c}\text { (gact)4g } \\
\text { CATGA } \\
\text { CAAAAA } \\
\text { CTAGC } \\
\text { CCC }\end{array}$ & 0.3 & 36 & $\mathrm{~F}$ & $\begin{array}{c}\text { C (yellow), } \\
\text { T (red) }\end{array}$ \\
\hline & $\mathrm{R}$ & $\begin{array}{c}\text { TGGAT } \\
\text { AAGAT } \\
\text { TGAGA } \\
\text { GAGT } \\
\text { GAGGA }\end{array}$ & 0.075 & & & & & & \\
\hline \multirow[t]{2}{*}{7146} & F & $\begin{array}{l}\text { AGACC } \\
\text { AAACC } \\
\text { TACGC } \\
\text { CAAAA }\end{array}$ & 0.15 & 130 & $\begin{array}{l}\text { ct(gact)cga } \\
\text { TACGC } \\
\text { CAAAA } \\
\text { TCCA } \\
\text { TTTC }\end{array}$ & 0.3 & 58 & F & $\begin{array}{l}\text { A (green), } \\
\text { G (blue) }\end{array}$ \\
\hline & $\mathrm{R}$ & $\begin{array}{c}\text { GGTGTA } \\
\text { TGCATC } \\
\text { GGGGTAGT }\end{array}$ & 0.15 & & & & & & \\
\hline \multirow[t]{2}{*}{10034} & F & $\begin{array}{c}\text { TCTCC } \\
\text { ATCTA } \\
\text { TTGATG } \\
\text { AGGGTCT }\end{array}$ & 0.3 & 108 & $\begin{array}{c}\text { ct(gact)4g } \\
\text { GTACC } \\
\text { GTTAAC } \\
\text { TTCCA } \\
\text { ATTAA } \\
\text { CTAG }\end{array}$ & 0.15 & 44 & $\mathrm{~F}$ & $\begin{array}{c}\text { C (yellow), } \\
\text { T (red) }\end{array}$ \\
\hline & $\mathrm{R}$ & $\begin{array}{c}\text { TTAAG } \\
\text { GCGAA } \\
\text { GTTTAT } \\
\text { TACTC } \\
\text { TTTTT }\end{array}$ & 0.3 & & & & & & \\
\hline 10238 & F & $\begin{array}{c}\text { GCGTCC } \\
\text { CTTTCT } \\
\text { CCATAAAA }\end{array}$ & 0.25 & 80 & $\begin{array}{c}\text { ct(gact) } 4 \text { gac } \\
\text { CTCCA } \\
\text { TAAAAT } \\
\text { TCTTCT } \\
\text { TAGTA } \\
\text { GCTAT }\end{array}$ & 0.3 & 48 & $\mathrm{~F}$ & $\begin{array}{c}\text { C (yellow), } \\
\text { T (red) }\end{array}$ \\
\hline
\end{tabular}




\begin{tabular}{|c|c|c|c|c|c|c|c|c|c|}
\hline & $\mathrm{R}$ & $\begin{array}{c}\text { GGGTA } \\
\text { AAAGG } \\
\text { AGGG } \\
\text { CAATTT }\end{array}$ & 0.25 & & & & & & \\
\hline 10400 & F & $\begin{array}{c}\text { GCCCTA } \\
\text { AGTCT } \\
\text { GGCCT } \\
\text { ATGA }\end{array}$ & 0.15 & 90 & $\begin{array}{c}\text { ct(gact)5gac } \\
\text { CGTTT } \\
\text { TGTT } \\
\text { TAAACT } \\
\text { ATATACC } \\
\text { AATTC }\end{array}$ & 0.3 & 52 & $\mathrm{R}$ & $\begin{array}{l}\mathrm{C} \text { (blue), } \mathrm{T} \\
\text { (green) }\end{array}$ \\
\hline & $\mathrm{R}$ & $\begin{array}{c}\text { TGAGTC } \\
\text { GAAATC } \\
\text { ATTCG } \\
\text { TTTT }\end{array}$ & 0.15 & & & & & & \\
\hline 12705 & F & $\begin{array}{c}\text { CCCAA } \\
\text { ACATTA } \\
\text { ATCAGT } \\
\text { TCTT } \\
\text { CAA }\end{array}$ & 0.18 & 102 & $\begin{array}{l}\text { t(gact)6g } \\
\text { TTAATC } \\
\text { AGTT } \\
\text { CTTCAA } \\
\text { ATATCT } \\
\text { ACTCAT }\end{array}$ & 0.15 & 54 & $\mathrm{~F}$ & $\begin{array}{c}\text { C (yellow), } \\
\text { T (red) }\end{array}$ \\
\hline & $\mathrm{R}$ & $\begin{array}{l}\text { TCTCA } \\
\text { GCCGA } \\
\text { TGAAC } \\
\text { AGTTG }\end{array}$ & 0.18 & & & & & & \\
\hline 12950 & F & $\begin{array}{c}\text { TCCTC } \\
\text { GCCTTA } \\
\text { GCATG } \\
\text { ATTT }\end{array}$ & 0.2 & 101 & $\begin{array}{l}\text { act(gact)n } \\
\text { TGAGG } \\
\text { CTTGGAT } \\
\text { TAGCG }\end{array}$ & 0.5 & 64 & $\mathrm{R}$ & $\begin{array}{c}\text { A (red), C } \\
\text { (blue), G } \\
\text { (yellow) }\end{array}$ \\
\hline & $\mathrm{R}$ & $\begin{array}{l}\text { GAGGC } \\
\text { CTAGT } \\
\text { AGTGG } \\
\text { GGTGA }\end{array}$ & 0.2 & & & & & & \\
\hline 13966 & F & $\begin{array}{c}\text { ACCGC } \\
\text { ACAATCC } \\
\text { CCTA } \\
\text { TCTA }\end{array}$ & 0.25 & 132 & $\begin{array}{c}\text { ct(gact) } 13 \\
\text { GCAGGT } \\
\text { TTTGG } \\
\text { CTCG }\end{array}$ & 0.5 & 69 & $\mathrm{R}$ & $\begin{array}{c}\text { A (red), G } \\
\text { (yellow) }\end{array}$ \\
\hline & $\mathrm{R}$ & $\begin{array}{c}\text { AGGTG } \\
\text { ATGATG } \\
\text { GAGGT } \\
\text { GGAG }\end{array}$ & 0.25 & & & & & & \\
\hline 15301 & F & $\begin{array}{c}\text { CСACCCTC } \\
\text { ACACGA } \\
\text { TTC } \\
\text { TTT }\end{array}$ & 0.08 & 119 & $\begin{array}{c}\text { ct(gact) } 4 \\
\text { ATTCTT } \\
\text { TACCT } \\
\text { TTCACT } \\
\text { TCATCTT }\end{array}$ & 0.15 & 42 & F & $\begin{array}{c}\text { A (green), } \\
\text { G (blue) }\end{array}$ \\
\hline & $\mathrm{R}$ & $\begin{array}{c}\text { GGTG } \\
\text { ATTCCTA } \\
\text { GGGGG } \\
\text { TTGT }\end{array}$ & 0.08 & & & & & & \\
\hline
\end{tabular}


Table 2: Multiplex 2Primers details

\begin{tabular}{|c|c|c|c|c|c|c|c|c|c|}
\hline \multirow[b]{2}{*}{ Site } & & \multicolumn{2}{|c|}{ PCR amplification } & \multirow[b]{2}{*}{$\begin{array}{l}\text { Amplicon } \\
\text { size (bp) }\end{array}$} & \multicolumn{2}{|c|}{ Single-base extension } & \multirow[b]{2}{*}{ Length } & \multirow[b]{2}{*}{ Orientation } & \multirow[b]{2}{*}{$\begin{array}{c}\text { Alleles } \\
\text { (dye) }\end{array}$} \\
\hline & & $\begin{array}{c}\text { Primer } \\
\text { sequences } \\
\left(5^{\prime}-3^{\prime}\right)\end{array}$ & $\begin{array}{l}\text { Concen } \\
\text { tration }\end{array}$ & & $\begin{array}{c}\text { Primer sequence } \\
\text { (5'-3') (5' aspecific } \\
\text { tail in lowercase } \\
\text { italics) }\end{array}$ & $\begin{array}{l}\text { Concen } \\
\text { tration }\end{array}$ & & & \\
\hline & & & $(\mathrm{pM})$ & & & $(\mathrm{pM})$ & (nt) & & \\
\hline \multirow[t]{2}{*}{2706} & $\mathrm{~F}$ & $\begin{array}{l}\text { CGAGG } \\
\text { GTTCAGC } \\
\text { TGTCT } \\
\text { CTT }\end{array}$ & 0.04 & 88 & $\begin{array}{l}\text { (gact)w } \\
\text { GTCTT } \\
\text { CTCGT } \\
\text { CTTGC } \\
\text { TGTGT }\end{array}$ & 0.1 & 60 & $\mathrm{R}$ & $\begin{array}{c}\text { A } \\
\text { (red), G } \\
\text { (yellow) }\end{array}$ \\
\hline & $\mathrm{R}$ & $\begin{array}{l}\text { AGGGT } \\
\text { CTTCTC } \\
\text { GTCTTG } \\
\text { CTG }\end{array}$ & 0.04 & & & & & & \\
\hline \multirow[t]{2}{*}{3348} & $\mathrm{~F}$ & $\begin{array}{c}\text { CAGTC } \\
\text { AGAGGTTC } \\
\text { AATTCC } \\
\text { TCTT }\end{array}$ & 0.2 & 142 & $\begin{array}{c}\text { act(gact),,g } \\
\text { GGAA } \\
\text { TGCCA } \\
\text { TTGC } \\
\text { GAT }\end{array}$ & 0.2 & 64 & $\mathrm{R}$ & $\begin{array}{c}\text { A } \\
\text { (red), G } \\
\text { (yellow) }\end{array}$ \\
\hline & $\mathrm{R}$ & $\begin{array}{l}\text { GGGCC } \\
\text { TTTGC } \\
\text { GTAGT } \\
\text { TGTAT }\end{array}$ & 0.2 & & & & & & \\
\hline \multirow[t]{2}{*}{3480} & $\mathrm{~F}$ & $\begin{array}{c}\text { CGCT } \\
\text { GACGC } \\
\text { CATAA } \\
\text { ААСTCT }\end{array}$ & 0.12 & 120 & $\begin{array}{c}\text { act(gact)2ga } \\
\text { GCCATA } \\
\text { AAACT } \\
\text { CTTC } \\
\text { ACCAA }\end{array}$ & 0.15 & 33 & $\mathrm{~F}$ & $\begin{array}{c}\text { A } \\
\text { (green), } \\
\text { G (blue) }\end{array}$ \\
\hline & $\mathrm{R}$ & $\begin{array}{l}\text { AGGGG } \\
\text { GGTTC } \\
\text { ATAGT } \\
\text { AGAAG }\end{array}$ & 0.12 & & & & & & \\
\hline 8281 & $\mathrm{F}$ & $\begin{array}{l}\text { GAAAT } \\
\text { CTGTGG } \\
\text { AGCA } \\
\text { AACCAC }\end{array}$ & 0.5 & $179 / 170$ & $\begin{array}{c}\text { (gact)2g } \\
\text { CCCTA } \\
\text { TAGCAC } \\
\text { CCCC } \\
\text { TCTA }\end{array}$ & 0.7 & 28 & $\mathrm{~F}$ & $\begin{array}{c}\text { a } \\
\text { (yellow), } \\
\text { d (blue)* }\end{array}$ \\
\hline \multicolumn{10}{|l|}{8289} \\
\hline \multirow{3}{*}{11251} & $\mathrm{R}$ & $\begin{array}{c}\text { AGAG } \\
\text { GTGTT } \\
\text { GGTTC } \\
\text { TCTTAA } \\
\text { TCTTT }\end{array}$ & 0.5 & & & & & & \\
\hline & $\mathrm{F}$ & $\begin{array}{l}\text { TGAA } \\
\text { CGCAGG } \\
\text { CACAT } \\
\text { ACTTC }\end{array}$ & 0.1 & 92 & $\begin{array}{l}\mathrm{t} \text { (gact)5gac } \\
\text { CCCCT } \\
\text { ACTCA } \\
\text { TCGCACT }\end{array}$ & 0.05 & 41 & $\mathrm{~F}$ & $\begin{array}{c}\text { A } \\
\text { (green), } \\
\text { G (blue) }\end{array}$ \\
\hline & $\mathrm{R}$ & $\begin{array}{c}\text { TGAGCC } \\
\text { TAGGGTG } \\
\text { TTGTGAG }\end{array}$ & 0.1 & & & & & & \\
\hline
\end{tabular}


International Journal of Cell Science \& Molecular Biology

\begin{tabular}{|c|c|c|c|c|c|c|c|c|c|}
\hline 11719 & $\mathrm{~F}$ & $\begin{array}{l}\text { GGCGCA } \\
\text { GTCAT } \\
\text { TCTCAT } \\
\text { AATC }\end{array}$ & 0.1 & 85 & $\begin{array}{c}\text { (gact)6 } \\
\text { GCAGA } \\
\text { ATAGTA } \\
\text { ATGAG } \\
\text { GATG } \\
\text { TAAG }\end{array}$ & 0.15 & 48 & $\mathrm{R}$ & $\begin{array}{c}\text { A } \\
\text { (red), G } \\
\text { (yellow) }\end{array}$ \\
\hline & $\mathrm{R}$ & $\begin{array}{c}\text { TGTGAG } \\
\text { TGCGTTC } \\
\text { GTAGTTTG }\end{array}$ & 0.1 & & & & & & \\
\hline \multirow[t]{2}{*}{12308} & $\mathrm{~F}$ & $\begin{array}{l}\text { CAGCT } \\
\text { ATCCAT } \\
\text { TGGTCT } \\
\text { TAGGC }\end{array}$ & 2 & 169 & $\begin{array}{c}\text { t(gact)9gac } \\
\text { TGGTC } \\
\text { TTAGG } \\
\text { CCCCAA }\end{array}$ & 3 & 56 & F & $\begin{array}{c}\text { A } \\
\text { (green), } \\
\text { G (blue) }\end{array}$ \\
\hline & $\mathrm{R}$ & $\begin{array}{l}\text { GATTTT } \\
\text { ACATA } \\
\text { ATGGG } \\
\text { GGTAT } \\
\text { GAGT }\end{array}$ & 2 & & & & & & \\
\hline \multirow[t]{2}{*}{12705} & $\mathrm{~F}$ & $\begin{array}{c}\text { ACTTC } \\
\text { TCCAT } \\
\text { AATATT } \\
\text { CATC } \\
\text { CCTGT }\end{array}$ & 1.3 & 184 & $\begin{array}{c}\text { act(gact)5g } \\
\text { TTAAT } \\
\text { CAGTT } \\
\text { CTTCAA } \\
\text { ATATC } \\
\text { TACTCAT } \\
\end{array}$ & 0.8 & 52 & F & $\begin{array}{c}\text { C } \\
\text { (yellow), } \\
\text { T (red) }\end{array}$ \\
\hline & $\mathrm{R}$ & $\begin{array}{c}\text { TCTCAGC } \\
\text { CGATGA } \\
\text { ACAGTTG }\end{array}$ & 1.3 & & & & & & \\
\hline \multirow[t]{2}{*}{13368} & $\mathrm{~F}$ & $\begin{array}{c}\text { CGCCTT } \\
\text { CTTCA } \\
\text { AAGCC } \\
\text { ATAC }\end{array}$ & 0.25 & 127 & $\begin{array}{c}\text { ct(gact)2gac } \\
\text { TAAGG } \\
\text { TTGTGG } \\
\text { ATGAT } \\
\text { GGA }\end{array}$ & 0.3 & 32 & $\mathrm{R}$ & $\begin{array}{c}\text { A } \\
\text { (red), G } \\
\text { (yellow) }\end{array}$ \\
\hline & $\mathrm{R}$ & $\begin{array}{c}\text { GGTGAG } \\
\text { GGAGGT } \\
\text { TGAA } \\
\text { GTGA }\end{array}$ & 0.25 & & & & & & \\
\hline \multirow[t]{2}{*}{13928} & $\mathrm{~F}$ & $\begin{array}{l}\text { CAGCC } \\
\text { CTAGA } \\
\text { CCTCAA } \\
\text { CTACCT }\end{array}$ & 0.04 & 119 & $\begin{array}{c}\text { ct(gact)5ga } \\
\text { AACAT } \\
\text { ACTCG } \\
\text { GATTCT } \\
\text { ACCCTA }\end{array}$ & 0.04 & 46 & F & $\begin{array}{c}\mathrm{C} \\
\text { (yellow), } \\
\mathrm{G} \text { (blue) }\end{array}$ \\
\hline & $\mathrm{R}$ & $\begin{array}{l}\text { ATAGGG } \\
\text { GATTGTG } \\
\text { CGGTGT }\end{array}$ & 0.04 & & & & & & \\
\hline \multirow[t]{2}{*}{14766} & $\mathrm{~F}$ & $\begin{array}{l}\text { TCAACT } \\
\text { ACAAGA } \\
\text { ACACCA } \\
\text { ATGACC }\end{array}$ & 0.05 & 109 & $\begin{array}{l}\text { c GACC } \\
\text { CCAA } \\
\text { TACG } \\
\text { CAAAA }\end{array}$ & 0.15 & 18 & F & $\begin{array}{c}\mathrm{C} \\
\text { (yellow), } \\
\text { T (red) }\end{array}$ \\
\hline & $\mathrm{R}$ & $\begin{array}{c}\text { ATCAT } \\
\text { GCGGAG } \\
\text { ATGTT } \\
\text { GGAT }\end{array}$ & 0.05 & & & & & & \\
\hline
\end{tabular}


International Journal of Cell Science \& Molecular Biology

\begin{tabular}{|c|c|c|c|c|c|c|c|c|c|}
\hline 15904 & F & $\begin{array}{c}\text { CATCC } \\
\text { GTACTA } \\
\text { TACTTC } \\
\text { ACAAC } \\
\text { AATC }\end{array}$ & 1 & 184 & $\begin{array}{c}\text { act(gact) } 4 \\
\text { GGCCTG } \\
\text { TCCTT } \\
\text { GTAGTA } \\
\text { TAAA }\end{array}$ & 0.6 & 40 & $\mathrm{~F}$ & $\begin{array}{c}\mathrm{C} \\
\text { (yellow), } \\
\text { T (red) }\end{array}$ \\
\hline & $\mathrm{R}$ & $\begin{array}{c}\text { GGTGCT } \\
\text { AATGGTG } \\
\text { GAGTT } \\
\text { AAAGA }\end{array}$ & 1 & & & & & & \\
\hline
\end{tabular}

Table 3: Mitochondrial haplogroups frequency in Isfahan province and some population

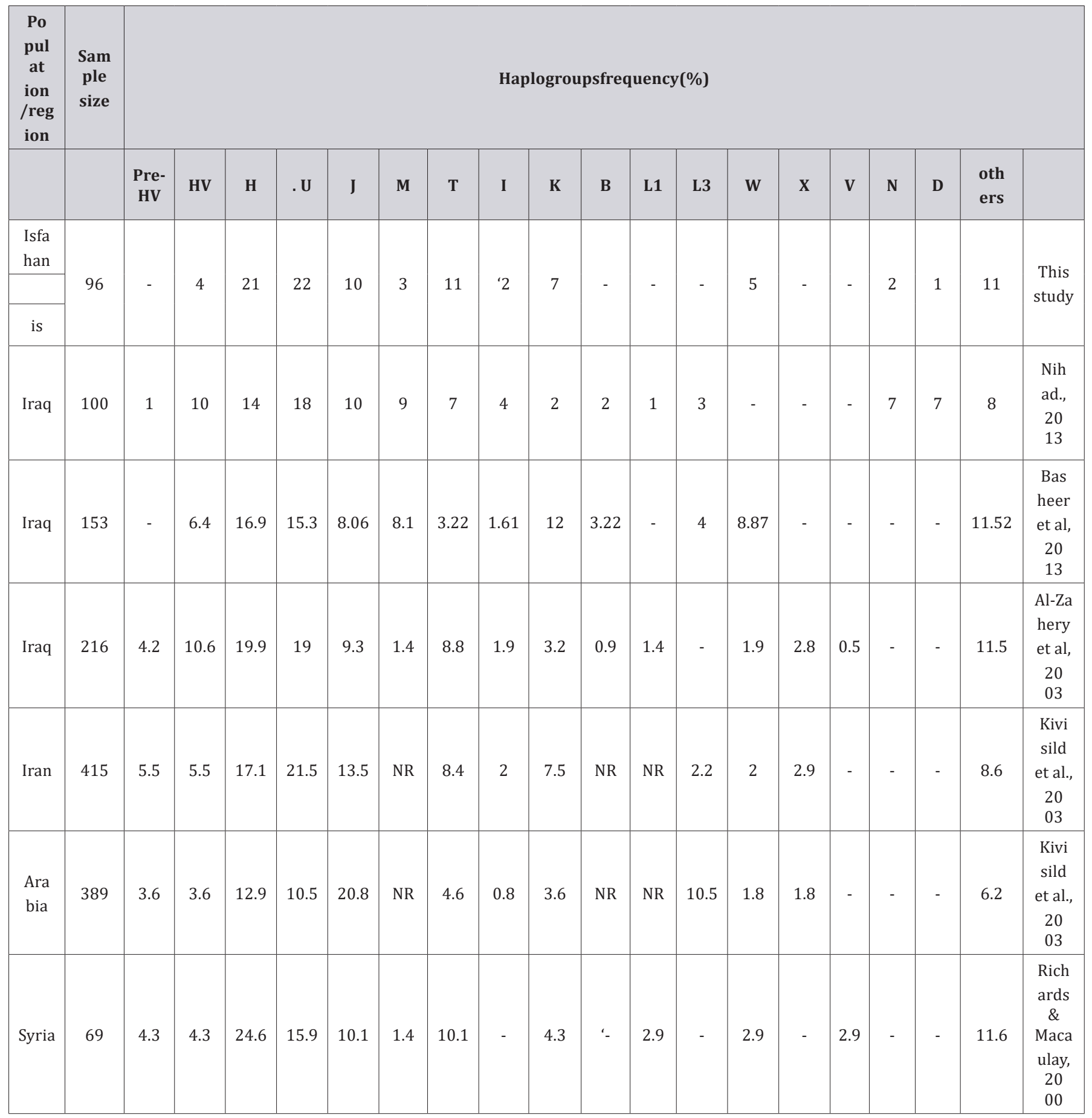




\begin{tabular}{|c|c|c|c|c|c|c|c|c|c|c|c|c|c|c|c|c|c|c|c|c|}
\hline $\begin{array}{c}\text { Pales } \\
\text { tin } \\
\text { ian }\end{array}$ & 117 & 1.7 & 1.7 & 30.8 & 7.6 & 9.4 & 1.7 & 12.8 & - & 6.8 & - & 0.9 & - & 2.6 & 3.4 & '- & - & - & 15.4 & $\begin{array}{c}\text { Rich } \\
\text { ards } \\
\& \\
\text { Mac } \\
\text { au } \\
\text { lay, } \\
2000\end{array}$ \\
\hline $\begin{array}{l}\text { Geor } \\
\text { gian }\end{array}$ & 139 & 7.2 & 7.2 & 17.3 & 21.6 & 3.6 & 2.9 & 12.9 & 2.2 & 10.1 & - & - & - & 1.4 & 10.1 & 0.7 & - & - & 9.3 & $\begin{array}{c}\text { Tam } \\
\text { bets } \\
\text { et } \\
\text { al, } \\
2000\end{array}$ \\
\hline $\begin{array}{l}\text { Ame } \\
\text { rican }\end{array}$ & 192 & 7.3 & 7.3 & 30.9 & 22.5 & 8.9 & $-\wedge$ & 11.5 & 1.6 & 7.9 & 0.5 & - & - & 1 & 2.1 & - & - & - & 5.3 & $\begin{array}{c}\text { Tam } \\
\text { bets } \\
\text { et al, } \\
20 \\
00\end{array}$ \\
\hline $\begin{array}{l}\text { Ana } \\
\text { tolia }\end{array}$ & 388 & 3.6 & 3.6 & 25 & 19.3 & 10.9 & 4.4 & 11.9 & 2.3 & 5.9 & & NR & 0.3 & 3.9 & 4.4 & - & - & - & 4.5 & $\begin{array}{c}\text { Kivi } \\
\text { sild } \\
\text { et al., } \\
20 \\
03 ; \\
\text { Tam } \\
\text { bets } \\
\text { et al, } \\
20 \\
00\end{array}$ \\
\hline $\begin{array}{l}\text { Ital } \\
\text { ian }\end{array}$ & 99 & 2 & 2 & 33.3 & 22.2 & 7.1 & '- & 9.1 & 4 & 8.1 & $-\mathrm{J}$ & - & - & 2 & 3 & 5.1 & - & - & 4.1 & $\begin{array}{c}\text { Tor } \\
\text { roni } \\
\text { et al, } \\
20 \\
03\end{array}$ \\
\hline Slav & 324 & NR & NR & 41.4 & 19.4 & 10.5 & 0.9 & 12.3 & 2.8 & 3.7 & ' - & - & - & 0.9 & 0.6 & 3.1 & - & - & 4.4 & $\begin{array}{c}\text { Kivis } \\
\text { ild } \\
\text { et al., } \\
19 \\
99 \\
\end{array}$ \\
\hline $\begin{array}{c}\text { Fin } \\
\text { no } \\
\text {-Ugri }\end{array}$ & 149 & NR & NR & 45.6 & 22.8 & 12.1 & 0.7 & 6 & 1.4 & 3.3 & - & - & - & 2.7 & 2 & 2 & - & - & 1.4 & $\begin{array}{c}\text { Kivisi } \\
\text { ld } \\
\text { et al., } \\
19 \\
99\end{array}$ \\
\hline $\begin{array}{l}\text { Ger } \\
\text { man }\end{array}$ & 200 & NR & NR & 50 & 13.5 & 7.5 & - & 8.5 & 2.5 & 6.5 & -5 & - & ' & 1 & 0.5 & 2.5 & - & - & 7.5 & $\begin{array}{c}\text { Lutz } \\
\text { et al., } \\
19 \\
98\end{array}$ \\
\hline $\begin{array}{c}\text { C-Asi } \\
\text { an }\end{array}$ & 205 & NR & NR & 14 & 8 & 2.5 & 38.5 & 3.5 & 1 & 0.5 & 6.8 & - & - & 1 & - & - & - & - & 24.2 & $\begin{array}{c}\text { Com } \\
\text { as } \\
\text { et al., } \\
19 \\
98\end{array}$ \\
\hline $\begin{array}{l}\text { Ind } \\
\text { ian }\end{array}$ & 1300 & 0.6 & 0.6 & 2.4 & 12 & 0.8 & NR & 1.1 & 0.6 & 0.2 & NR & - & - & 1.5 & 0.2 & - & - & - & 13.8 & $\begin{array}{c}\text { Kivis } \\
\text { ild } \\
\text { et al., } \\
20 \\
03\end{array}$ \\
\hline
\end{tabular}

Multiplex PCRs were performed in a reaction volume of $6 \mu \mathrm{l}, \quad 0.35$ units of Taq DNA polymerase, $1.2 \mu \mathrm{l}$ watery genomic DNA in which contained $1 \mathrm{X}$ Gene Amp PCR buffer, $100 \mu \mathrm{M}$ of each dNTPs $\quad 5 \mathrm{mg} / \mathrm{ml}$ and 15 pairs PCR primers for multiplex1 (Table1) and 12 
pairs PCR primers for multiplex 2 (Table 2). The reactions were performed in a ABI Bio system applied 2730 with conditions: an early denaturation at $95{ }^{\circ} \mathrm{C}$ for 10 minuet and 30 cycles of 94 ${ }^{\circ} \mathrm{C}$ for 15 seconds, $60{ }^{\circ} \mathrm{C}$ for 45 seconds and a final extension at $60{ }^{\circ} \mathrm{C}$ for 5 minuet. Then PCR products were purified by adding $1.5 \mu \mathrm{l}$ ExoSAP to them and incubation at $37^{\circ} \mathrm{C}$ for 15 minuet and $80{ }^{\circ} \mathrm{C}$ for 15 minute. Multiplex SNaPShot were performed in a reaction volume of $5 \mu \mathrm{l}$, which contained $1 \mu \mathrm{l}$ Ready Reaction Mix, $1 \mu \mathrm{l}$ purified PCR product and extensions primers (Table1 \& 2). The reactions were performed in conditions: $96^{\circ} \mathrm{C}$ for 2 minutes and 25 cycles of $96^{\circ} \mathrm{C}$ for 10 seconds, $50{ }^{\circ} \mathrm{C}$ for 5 seconds and 60 Results o $C$ for 30 seconds. The reaction products were purified by adding $1 \mu \mathrm{l}$ CIP to them and incubation at $37 \stackrel{\circ}{\circ} \mathrm{C}$ for 45 minutes and 75 ${ }^{\circ} \mathrm{C}$ for 15 minutes. prepared a mixture of $1 \mu \mathrm{l}$ purified extension product, $8.8 \mu \mathrm{l} \mathrm{Hi}$ - Di for maimed and $0.2 \mu \mathrm{l}$ GeneScan-120 LIZ size standard and ran samples in genetic analyzer ABI 1330XL with POP-7 polymer. Results were analyzed using Gene Mapper ID version 3.2 software. The results of SNP patterns in two assays was determining mitochondrial haplo groups in samples of the study. Then for support of our detective haplo groups by SNaPShot system, D-Loop region was sequenced in some samples by genetic analyzer ABI 1330XL.

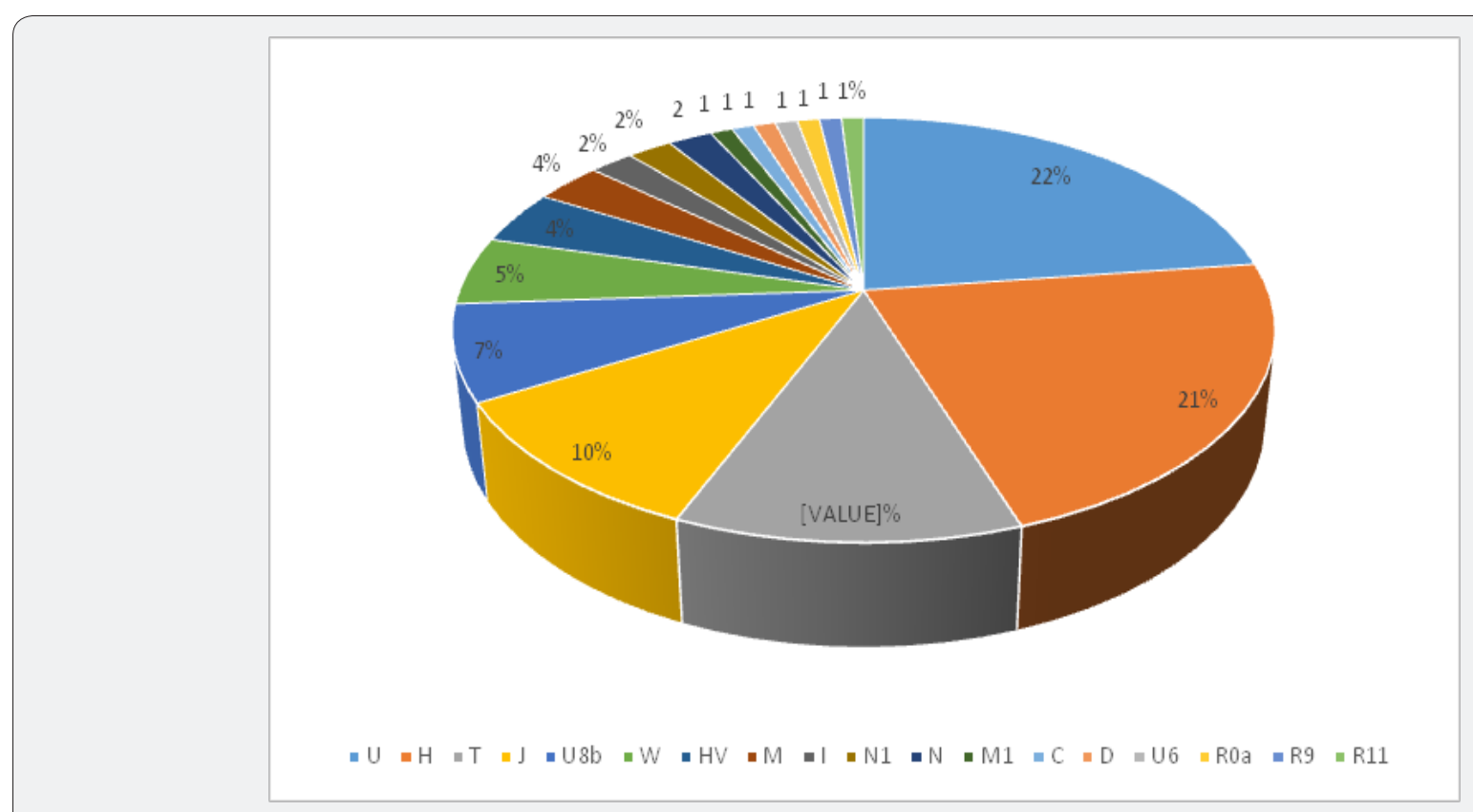

Figure 2: Mitochondrial DNA haplogroups frequency (\%) in a selective population of Isfahan province..

$96 \mathrm{mtDNA}$ samples from Isfahan province were analyzed and 18 haplogroups were found (Figure 2). 94\% of this population belonged to Western Eurasian haplo groups and only 5\% belonged to Eastern Eurasian haplo groups (C, D, R9, R11, M and N). So Western Eurasian haplo groups were predominant. Haplo group $\mathrm{U}$ and $\mathrm{H}(22 \%, 21 \%)$ and then T, J and U8b (11\%, 10\% and $7 \%$ ) had the most frequency and Eastern Eurasian haplo groups and a North African haplo group (M1) were present at a lower frequency.

\section{Discussion}

Previous studies indicated a high frequency of $77 \%$ of Asian mtDNA is belonged to haplogroup M. Since Iran is located in Asia it is expected that this haplo group has a high frequency in Iran. But results of Houshmand et al. 2004 study showed a low frequency of this haplo group (0-4\%). This study found the same results and only three cases of persons who take part in this study had haplo group M.

In other side about 99\% of mtDNA in European population belong to at least one of the nine haplogroups: $\mathrm{H}, \mathrm{U}, \mathrm{J}, \mathrm{T}, \mathrm{K}$,
I, V, W, and X. Six of them (H, J, T, K, I and w) are especial for European population and probably originated from Caucasoid and genetically separated from ancestors of African and Asian [7]. Studies about Iran country showed a high frequency of Haplo group U, also in this study had the most frequency (22\%). This haplo group is much older than the others and estimated to originate about 51,000-67,000 years ago [7]. It seems that this haplo group originated from Africa and then expanded into Middle East and Europe. In the other hand haplo groups $\mathrm{H}, \mathrm{T}$, $\mathrm{J}$ and $\mathrm{K}$ had the most frequency after haplo group $\mathrm{U}$ in Isfahan province. It is estimated that originated about $8,000-30,000$ years ago $[7,8]$.

\section{References}

1. Gilles RE, Blanc H, Cann HM, Wallace DC (1980) Maternal inheritance of human mitochondrial DNA. Proc Natl Acad Sci USA 77: 6715-6719.

2. Beltinger (2000) Mitochondrial amplification of death signals determines thymidine kinase/ ganciclovir-triggered activation of apoptosis. Cancer research 60: 12-17.

3. Stoneking M,Soodyall $H$ (1996) Human evolution and the mitochondrial genome. Curr Opin Genet Dev 6: 731 -736. 
4. Budowle B, Allard M, Wilson MR, Chakra borty R (2003) Forensics and mitochondrial DNA: Applications, Debates, and Foundations. Annu Rev Genomics Hum Genet 4: 119-141.

5. Saremi Mohammad Ali, Saremi Mahnaz, Tavallaei Mahmood (2008) Rapid genomic DNA extraction (RGDE). Forensic Science International: Genetics Supplement Series 1: 63-65.

6. Mannis van Oven, Mark Vermeulen, Manfred Kayser (2011) Multiplex genotyping system for efficient inference of matrilineal genetic ancestry with continental resolution. Investigative Genetics 2: 6.
7. Torroni A, Huoponen K, Francalacci P, Petrozzi M, Morelli L, et al. (1996) Classification of European mtDNAs from an analysis of three European populations. Genetics 144: 1835-1850.

8. Torroni A, Lott MT, Cabell MF, Chen YS, Lavergne L, et al. (1994) mtDNA and the origin of Caucasians: identification of ancient Caucasianspecific haplogroups, one of which is prone to a recurrent somatic duplication in the D-loop region. Am J Hum Genet 55: 760-776.

\section{Your next submission with Juniper Publishers will reach you the below assets}

- Quality Editorial service

- Swift Peer Review

- Reprints availability

- E-prints Service

- Manuscript Podcast for convenient understanding

- Global attainment for your research

- Manuscript accessibility in different formats ( Pdf, E-pub, Full Text, Audio)

- Unceasing customer service

Track the below URL for one-step submission https://juniperpublishers.com/online-submission.php 